\title{
Acupressure Therapy
}

National Cancer Institute

\section{Source}

National Cancer Institute. Acupressure Therapy. NCI Thesaurus. Code C15606.

The application of non-invasive finger pressure along energy points throughout the body in order to relieve pain and induce a feeling of well-being. 